\title{
3'-End formation of mouse pre-rRNA involves both transcription termination and a specific processing reaction
}

\author{
Anne Kuhn and Ingrid Grummt ${ }^{1}$ \\ Institut für Biochemie, Röntgenring 11, 8700 Würzburg, FRG
}

\begin{abstract}
We have studied the sequence requirements for 3 '-end formation of rDNA transcripts in a cell-free system and show that the generation of correct ends of mouse pre-rRNA is brought about by a two-step process that involves a bona fide termination reaction, followed by a specific trimming of the primary transcript by 10 nucleotides. We show that termination of mouse ribosomal gene transcription by RNA polymerase I (pol I) takes place in front of an 18-bp DNA sequence element (the 'Sal box'), which was previously shown to function as termination signal. Termination of pol I transcription occurs at a fixed distance (11 bp) upstream of the Sal box, independent of the sequence of adjacent gene regions. The processing reaction, however, is strongly influenced by sequences flanking the termination signal at the $5^{\prime}$ site. Substitution of a cluster of $T$ residues by guanines within the region of $3^{\prime}$-end formation abolishes the $3^{\prime}$-terminal trimming of the primary transcript. Interestingly, this $3^{\prime}$-terminal processing event, which can be uncoupled from the termination reaction, requires both a correct $3^{\prime}$ end and specific sequences in the $3^{\prime}$-terminal region of the primary transcript. Read-through transcripts generated in the extract system or by SP6 RNA polymerase are no substrate for the processing nuclease(s). Because the termination and processing activity can be separated chromatographically, the nucleolytic activity does not reside in TTF-I, the factor that binds to the Sal box and directs transcription termination.
\end{abstract}

[Key Words: Transcription termination; processing; RNA polymerase $I_{;} 3^{\prime}$-end formation]

Received August 8, 1988; revised version accepted December 1, 1988.

Of the various steps in eukaryotic transcription-initiation, elongation, and termination-probably least is known about the termination reaction, in part because each of the three nuclear RNA polymerases terminates transcription in a different manner. Termination by polymerase III (pol III) may be simple, occurring within clusters of four or more $\mathrm{T}$ residues, often surrounded by GC-rich sequences (Bogenhagen and Brown 1981; Cozzarelli et al. 1983). Mature tRNAs are generated by processing at both the $5^{\prime}$ and $3^{\prime}$ ends of the primary transcripts (Castano et al. 1985; Frendewey et al. 1985). Termination of mRNA coding genes, on the other hand, is heterogeneous and may occur far downstream of the mature $3^{\prime}$ end of the transcript. It has been shown that the 3 ' termini of most, if not all, mRNAs are generated by an RNA processing event that entails an endonucleolytic cleavage of the primary transcript 10-30 nucleotides downstream from the polyadenylation signal AATAAA (for a review, see Nevins 1983; Birnstiel et al. 1985). More recent studies demonstrated that a functional poly $(\mathrm{A})$ addition signal is a crucial element for RNA polymerase II (pol II) transcription termination

${ }^{1}$ Corresponding author.
(Whitelaw and Proudfoot 1986; Logan et al. 1987) and that both the GT-rich downstream element and the AATAAA conserved sequence are required for transcription termination by RNA polymerase II /Connelly and Manley 1988).

More is known about the molecular mechanisms directing termination of rDNA transcription by RNA polymerase I (pol I). In Xenopus laevis (Labhard and Reeder 1986, 1987), in mouse (Grummt et al. 1985, 1986), and in human rDNA (Bartsch et al. 1987), the sequences that are functionally important for the stop of the elongation reaction and the release of the nascent RNA chains have been identified. In the mouse system it has been shown that the $3^{\prime}$ end of pre-rRNA maps at a defined point 565 nucleotides downstream of the $28 \mathrm{~S}$ RNA-coding region (Grummt et al. 1985) in front of an 18-bp sequence motif AGGTCGACCAGA/TT/ ANTCCG (the Sal box). The 'Sal box' exerts its function as termination signal by interacting with a specific DNA-binding protein. Deletions, insertions, or point mutations within this sequence element decrease or eliminate factor binding and similarly inhibit or abolish transcription termination (Grummt et al. 1986). An 18-bp synthetic oligonucleotide encompassing the Sal box sequence has been shown to mediate both the stop of the elongation reaction and the release of the nascent 
RNA chains. In addition, gene regions flanking the Sal box at both the $5^{\prime}$ and $3^{\prime}$ site appear to affect both the efficiency and the position of $3^{\prime}$-end formation. We have shown recently that sequences downstream of the Sal box augment the efficiency of the termination reaction, whereas deletion of the gene region upstream of the box results in the formation of transcripts whose ends map 10 nucleotides farther downstream (Kuhn et al. 1988).

In this communication we report on the effect of sequences upstream of the termination signal on the generation of correct $3^{\prime}$ ends of pol I transcripts. We demonstrate that the end of mouse pre-rRNA is generated by a specific termination event, followed by a subsequent limited 3 '-terminal processing reaction. This processing step, which removes only 10 nucleotides from the primary transcript, requires a stretch of uridines in the RNA and is dependent on correct transcription termination.

\section{Results}

Two $3^{\prime}$ termini are detected in rDNA transcripts synthesized in vivo and in vitro

Recently, we have used a cell-free transcription system and a series of artificial minigenes that represent gene fusions between the mouse rDNA promoter and $3^{\prime}$-terminal spacer sequences to demonstrate the essential role of the 18-bp Sal box sequence element in termination of pol I transcription (Grummt et al. 1985, 1986; Bartsch et al. 1987; Kuhn et al. 1988). We have shown that sequences downstream of the Sal box augment the efficiency of $3^{\prime}$-end formation, whereas $5^{\prime}$-flanking sequences appear to affect the position of $3^{\prime}$ ends (Kuhn et al. 1988). To investigate this effect of adjacent gene sequences also in vivo, two artificial ribosomal minigenes (pMrC552-604 and pMrC552-650, see Fig. 1A) were constructed. In addition to 5 '- and 3 '-terminal rDNA fragments, these constructs contain part of the bacterial marker gene coding for chloramphenicol acetyltransferase (CAT) that has been positioned between the promoter and terminator region to allow the detection of pol I-specific transcripts above the high level of cellular pre-rRNA. The plasmids were used both as templates in the cell-free transcription system or to transfect $3 \mathrm{~T} 6$ cells. The RNA synthesized either in vitro or in vivo was analyzed by nuclease S1 mapping. As shown in Figure 1B (lanes 1, 2) RNA synthesized in the cell-free system yields a 188-nucleotide protected band that maps the $3^{\prime}$ ends of transcripts at nucleotide +565 relative to the end of 28S rRNA. This 3 ' terminus of rDNA transcripts, i.e., $21 \mathrm{bp}$ upstream of the Sal box, is generally formed in vitro by nuclear extracts and corresponds to the majority of $3^{\prime}$ ends of cellular pre-rRNA (Grummt et al. 1985). Surprisingly, the transcripts derived from the transfected cells (lanes 3,4) show an additional band (198 nucleotides) that maps 10 nucleotides closer to the termination signal, the Sal box. The presence of these longer transcripts in transfected cells could mean (1) that pol I stops transcription at two distinct sites that are differently
A

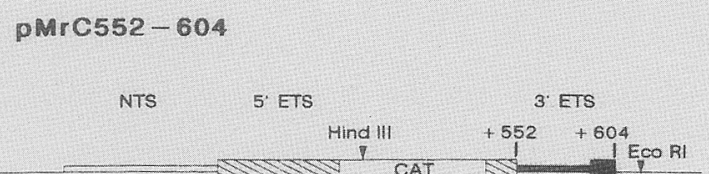

pMrC552-650

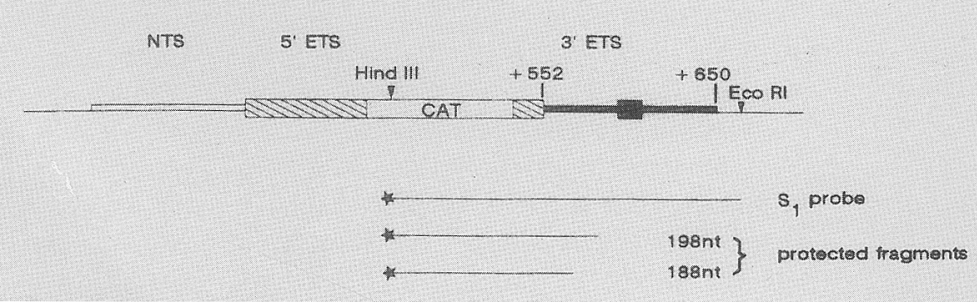

B

12 G A+C $3 \quad 4$
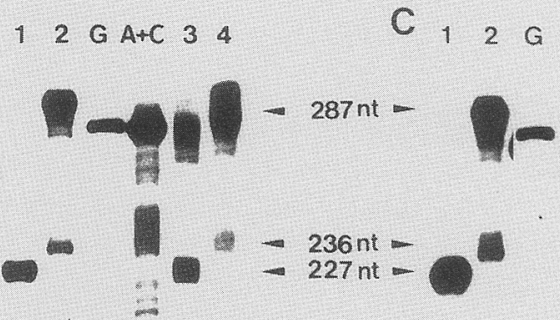

$\therefore$

s.
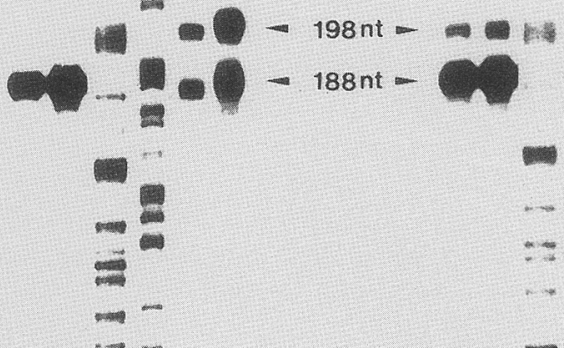

Figure 1. $3^{\prime}$ End mapping of transcripts synthesized in vitro or in vivo from ribosomal minigene constructs. $(A)$ Diagram of the plasmids pMrC552-604 and pMrC552-650. The black box marks the 18-bp Sal box element; the hatched regions represent sequences from the $5^{\prime}$ external transcribed spacer (ETS). The numbers refer to the position of 3 ' terminal sequences relative to the end of 285 RNA. (B) Plasmids pMrC552-604 (lanes 1,3) and pMrC552-650 (lanes 2,4) were transcribed for 60 min in the cell-free system (lanes 1,2 ) or were transfected into 3 T6 cells (lanes 3,4 ). The RNA was hybridized to the HindIII-EcoRI fragment derived from pMrC552$650(A)$ and treated with nuclease S1, and the protected fragments were analyzed on $6 \%$ sequencing gels, along with G- and AC-specific cleavage reactions. The 227-nucleotide bands in lanes 1 and 3 and the 287-nucleotide bands in lanes 2 and 4 represent readthrough transcripts; the extra 236-bp band is a nuclease S1 artifact due to cutting within the long stretch of $\mathrm{T}$ residues from +613 to +628 . $(C)$ The same reactions occurred as in lanes 1 and $2(B)$, except that the transcription was performed with another extract preparation and the reaction proceeded for only $30 \mathrm{~min}$. 
used in vivo and in vitro, or (2) that the actual termination process takes place at nucleotide +575 and the last 10 nucleotides are removed by nucleolytic cleavage. To distinguish between these two possibilities, conditions were established to produce these longer transcripts in vitro. As shown in Figure 1C, mixing of nuclear with S-100 extracts yielded transcripts with an identical S1 protection pattern as the RNA derived from the transfected cells. If transcription complexes were formed by preincubating the template DNA with extract proteins in the absence of nucleotides and the synthesis time was sufficiently short, the long transcripts were observed almost exclusively.

To find out whether the shorter molecules arise by $3^{\prime}$ terminal processing of the longer primary transcripts, RNA synthesis in vitro was stopped after $10 \mathrm{~min}$ by addition of actinomycin $\mathrm{D}$, and the incubation was continued for different periods of time. The analysis of the RNA by nuclease S1 mapping of the $3^{\prime}$ termini (Fig. $2 A, B \mid$ reveals a gradual decrease of the 198-nucleotide band and a concomitant increase of the 188-nucleotide band. This result indicates that there is a precursorproduct relationship between both RNA species and suggests that the trimming of the primary transcript by 10 nucleotides occurs both in vitro and in vivo.

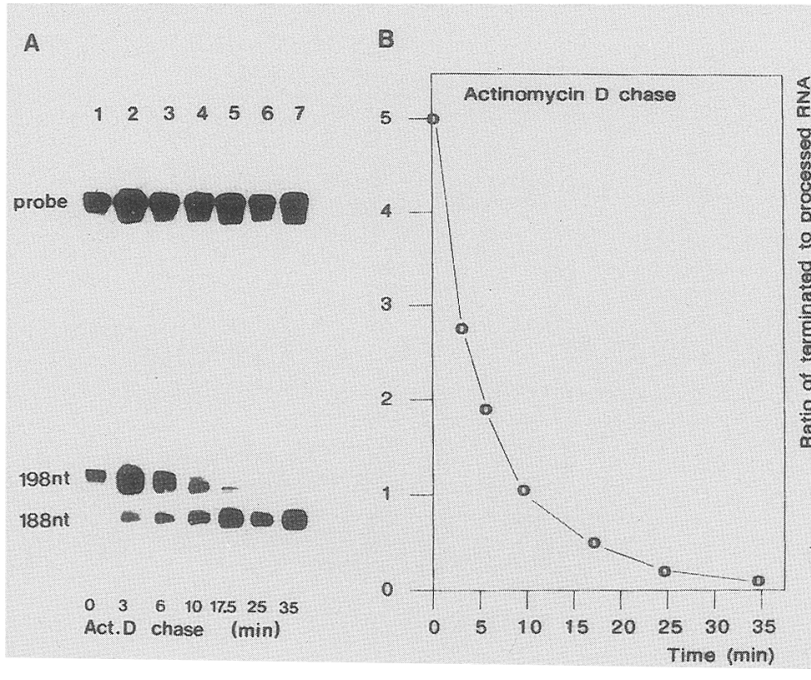

Figure 2. Mapping of $3^{\prime}$ termini of rDNA transcripts after actinomycin D chase. (A) A 200- $\mu$ l transcription assay containing $120 \mu \mathrm{g}$ of pMrC552-650 template DNA was preincubated for 15 min at $30^{\circ} \mathrm{C}$ under standard transcription conditions in the absence of NTPs. After addition of the nucleotides, transcription was allowed to proceed for $10 \mathrm{~min}$. RNA synthesis was stopped by addition of actinomycin $\mathrm{D}(20 \mu \mathrm{g} / \mathrm{ml})$, and the mixture was incubated further for $0,3,6,10,17.5,25$, and $35 \mathrm{~min}$, respectively (lanes $1-7)$. The RNA was isolated and mapped by the nuclease S1 technique. $(B)$ The terminated (198-nucleotide band) and the processed (188-nucleotide band) transcripts were quantitated by densitometry and the ratio of terminated vs. processed RNA was plotted against the time of the actinomycin D chase.

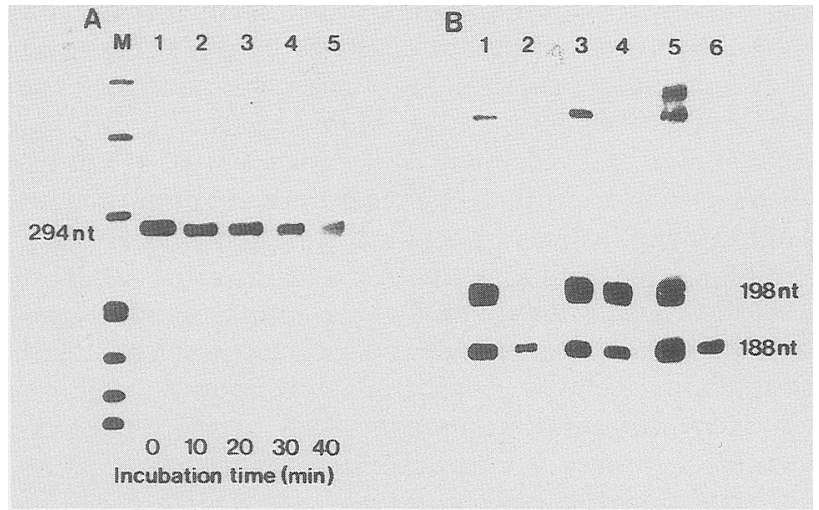

Figure 3. Uncoupling of the transcription process from the $3^{\prime}$ terminal processing reaction. (A) Plasmid DNA Sp64/C552650 was transcribed with SP6 polymerase, and the isolated transcripts were incubated under standard conditions in the cell-free system containing a mixture of S-100 and nuclear extract. After the times indicated, the RNA was extracted with phenol-chloroform and analyzed on a $6 \%$ sequencing gel, along with pBR322/HpaII size marker fragments. (B) Template DNA pMrC552-650 (15 ng) was preincubated in 25- $\mu$ l standard transcription reactions with extract protein in the absence of NTPs to allow the formation of transcription initiation complexes. Transcripts that were synthesized within a 10-min incubation period were isolated, and the template DNA was digested by treatment with DNase. The isolated RNA was either analyzed by the nuclease $S 1$ technique without further incubation (lanes 1,3,5) or after incubation for $20 \mathrm{~min}$ under standard conditions. The cleavage reaction was performed in the presence of extract protein (lanes 2,6), in the absence of extract protein (lane 4), or in the absence of NTPs (lane 6). The 198-nucleotide nuclease S1-resistant fragment corresponds to terminated primary transcripts; the 188-nucleotide fragment to 3 '-terminal processed RNA molecules.

\section{The 3'-terminal processing reaction requires correctly terminated RNA chains}

The next two experiments were carried out to determine whether the nucleolytic cleavage of pre-rRNA is coupled to the termination process or whether read-through RNA is also a substrate for the processing nuclease. For this, transcripts synthesized by SP6 polymerase from the plasmid SP64/C552-650 were incubated for different times in the extract system and analyzed by electrophoresis on denaturing gels. Figure $3 \mathrm{~A}$ shows the result of this experiment. Although there is some decrease in the total amount of RNA during prolonged incubation (which may be caused by low amounts of RNase activity present in the extracts), the size of the input RNA remains essentially unchanged, indicating that readthrough transcripts are not cleaved by the nuclease(s) responsible for the $3^{\prime}$-terminal processing of pre-rRNA. This result predicts that either the processing reaction is directly coupled to the termination process or the correct $3^{\prime}$ end of the primary transcript is recognized by the processing enzyme(s).

To test this prediction, we synthesized faithfully terminated transcripts in the cell-free system, isolated the RNA, added it back to the extract, and incubated the 
mixture for 20 min under different experimental conditions. As shown in Figure 3B (lane 1), the nuclease S1 analysis of the transcripts synthesized during the 15-min transcription period reveals an approximately equal ratio of terminated (198-nucleotide fragment) and processed (188-nucleotide fragment) transcripts. After incubation of the isolated transcripts with extract protein, only the 188-bp protected band is visible (lane 2), indicating that the terminated transcripts have been processed accurately. This finding suggests that the $3^{\prime}$-terminal trimming of the primary transcripts occurs post-transcriptionally and that only correctly terminated RNA molecules are substrates for the processing enzyme(s).

To investigate whether the processing reaction requires extract proteins or nucleotides, the isolated RNA was incubated in the cell-free system in the absence of either cell extract (Fig. 3, lanes 3, 4) or nucleoside triphosphates (NTPs) (lanes 5, 6). Clearly, in the absence of extract protein the ratio of terminated vs. read-through transcripts remains unchanged. The absence of nucleotides, on the other hand, does not affect the processing reaction. This requirement for cellular component|s| present in the cell extracts and the dispensability of nucleotides suggest that the 3 '-terminal processing event is not an autocatalytic reaction.

\section{Highly purified termination factor TTF-I does not possess nucleolytic activity}

The tight coupling of 3 '-terminal processing with the termination reaction raises the question whether the termination factor TTF-I that binds to the Sal box motif not only catalyzes the stop of the pol I elongation reaction and the release of the nascent RNA but is also responsible for the removal of the last 10 nucleotides from the primary transcript. For this, terminated transcripts were synthesized in a short-term reaction, were isolated, and incubated in the presence of highly purified factor TTF-I. As shown in Figure 4, TTF-I that has been purified to molecular homogeneity by sequence-specific affinity chromatography (Bartsch et al. 1988) does not convert the primary transcripts into processed molecules. This result indicates that transcription termination and $3^{\prime}$-terminal processing is brought about by different proteins.

\section{A cluster of thymidines is involved in the processing reaction}

To define which sequences control this $3^{\prime}$-processing activity, two mutants were constructed that contained specific nucleotide exchanges in the region removed from the primary transcript. As shown in Figure 5A, both mutants are analogous to the wild-type template pMrC552-690*. In mutant $\mathrm{pMrF1}$, the thymidine residues at positions $566-571$ have been converted into guanosines; in $\mathrm{pMrF2}$, the cytidine residues at positions $572-575$ were substituted by adenosines. Furthermore, a mutant (pMrF3) was constructed where all the 3 '-terminal spacer sequences upstream of the processed region

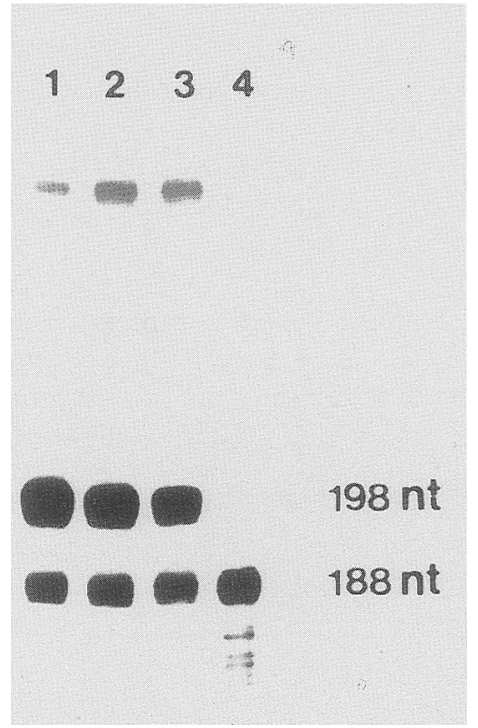

Figure 4. Purified transcription termination factor TTF-I does not possess nucleolytic activity. A $200 \mu$ l reaction mixture containing $120 \mathrm{ng}$ of pMrC552-650 template DNA was incubated with extract protein for $10 \mathrm{~min}$ at $30^{\circ} \mathrm{C}$ before transcription was initiated by addition of NTPs. After $10 \mathrm{~min}$ the nucleic acids were extracted and the DNA digested with DNase I. The isolated RNA was dissolved in $200 \mu \mathrm{l}$ of transcription buffer and incubated in $50-\mu \mathrm{l}$ aliquots for $0 \mathrm{~min}$ (lane 1) or 20 min (lanes 2-4), respectively. The reactions contained either 15 $\mu \mathrm{l}$ of buffer $\mathrm{BC}_{100}$ (lanes 1,2), $15 \mu \mathrm{l}$ of highly purified factor TTF-I (lane 3), or $15 \mu \mathrm{l}$ of cell extract (lane 4). The RNA was isolated and analyzed by S1 mapping.

were missing. In addition, 3 out of the 10 bases that were removed from the pre-rRNA during the processing reaction were deleted. The templates were assayed both for specific termination and for 3'-terminal processing. Wild-type and mutant templates were transcribed in the extract system for $10 \mathrm{~min}$, and the reactions were either stopped immediately (Fig. 5B, lanes 1, 3,5) or incubation was continued for another $20 \mathrm{~min}$ in the presence of actinomycin D (lanes 2, 4, 6) before RNA extraction and S1 analysis. In each case, the short-time transcription reactions reveal correctly terminated RNA molecules, indicating that the termination reaction is independent of 5 '-flanking sequences. After the actinomycin D chase, the conversion of the 198 nucleotides into the 188-nucleotide protected band is observed both in pMrC552$690^{*}$ and in mutant $\mathrm{pMrF} 2$, indicating that the $\mathrm{C}$ residues do not appear to play an essential role in the trimming reaction. However, transcripts derived from mutant pMrF1 fail to be processed specifically. This result suggests that the stretch of uridines present in the primary transcript between nucleotides +566 and +571 serves an important function in the processing reaction. Surprisingly, transcripts from the mutant template pMrF3 are specifically processed at the boundary between the $T$ cluster and the neighboring foreign DNA sequences, even though an additional three bases were deleted between the termination and the processing site. 


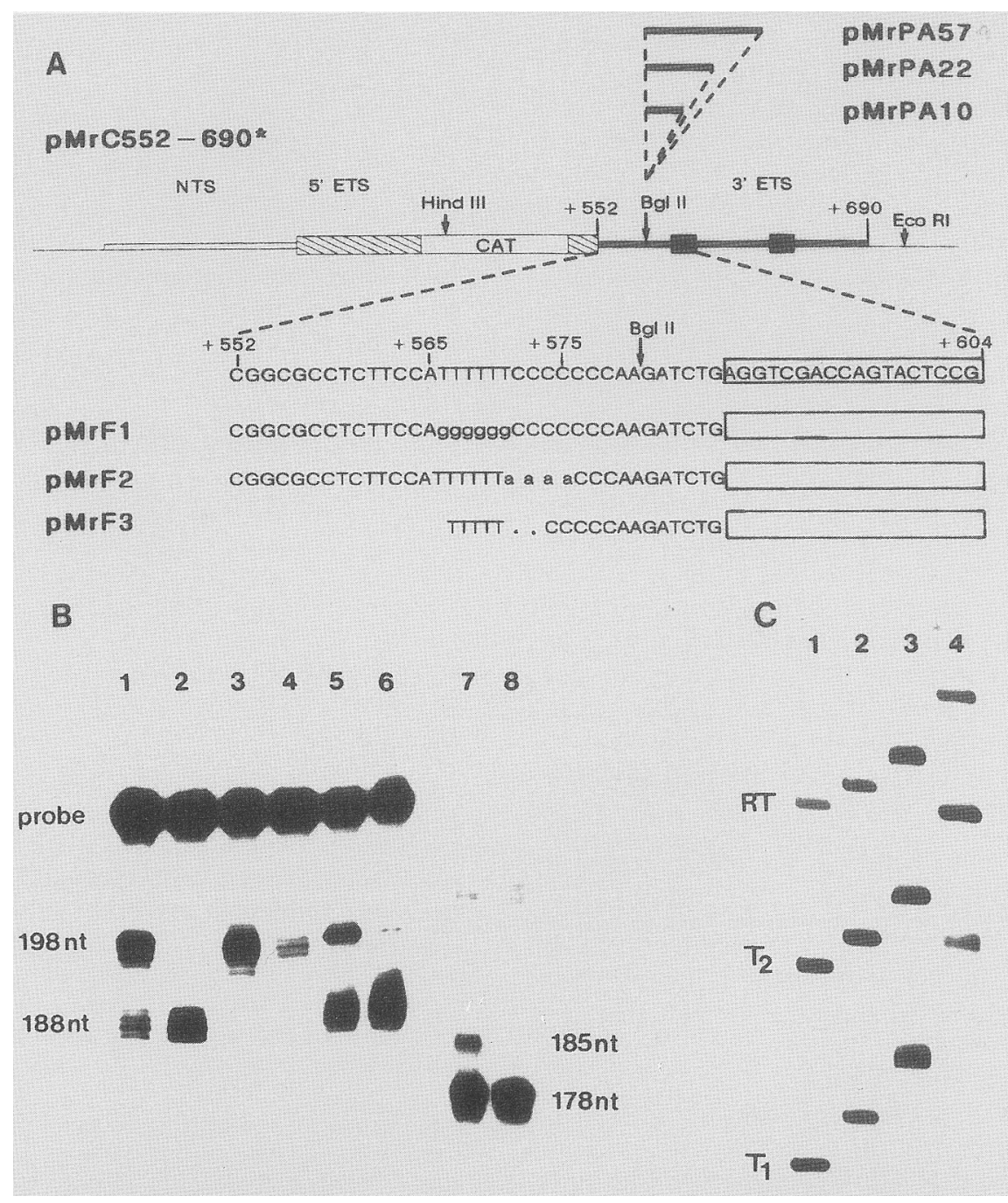

Figure 5. Effect of sequence alterations on termination and $3^{\prime}$-terminal processing. $(A \mid$ Schematic diagram of the mutants analyzed. The mutants containing base substitutions or deletions, respectively, in the $5^{\prime}$-flanking region (pMrF1, pMrF2, and pMrF3) and the push-apart mutants pMrPA10, pMrPA22, and pMrPA57 are derivatives of pMrC552-690*. The insertion of different DNA fragments into the unique BgllI site is shown above the scheme of the construct. The nucleotide sequence of the region preceding the first termination signal in the mutants $\mathrm{pMrF1}, \mathrm{pMrF2}$, and $\mathrm{pMrF3}$ is shown below. Exchanged nucleotides are indicated by lowercase letters; deleted nucleotides are marked by a dot. The 18-bp sequence encompassing the Sal box termination signal is boxed. $(B)$ Nuclease S1 mapping of transcripts derived from the mutants pMrF1, pMrF2, and pMrF3. The template DNAs pMrC552-590* (lanes 1,2 ), pMrF1 (lanes 3,4), pMrF2 (lanes 5,6), and pMrF3 (lanes 7,8) were preincubated with extract proteins for 15 min before transcription was started by addition of the NTPs. After $10 \mathrm{~min}$, the reaction was either stopped (lanes 1,3,5,7) or incubated for another $20 \mathrm{~min}$ in the presence of $20 \mu \mathrm{g} / \mathrm{ml}$ actinomycin D (lanes 2,4,6,8). The numbers mark the specific nuclease S1-resistant fragments that correspond to terminated (upper band) and processed (lower band) transcripts. (C) Transcripts generated from pMrC552-690* (lane 1) and the push-apart mutants pMrPA10 (lane 2), pMrPA22 (lane 3), and pMrPA57 (lane 4). Autoradiogram of transcripts synthesized from linear templates (digested with EcoRI) for $30 \mathrm{~min}$ at $30^{\circ} \mathrm{C}$ followed by a 20 -min incubation in the presence of actinomycin D. The lower and middle bands represent transcripts terminated at the first and second box, respectively. The upper bands are read-through transcripts.

From this result, we conclude that the specificity of the trimming reaction resides exclusively within the last 10 nucleotides, presumably within the stretch of uridines.

\section{$3^{\prime}$-Terminal processing requires the correct location of the Sal box element}

The results presented above suggest that transcription termination and the removal of the last 10 nucleotides of the primary transcript occur sequentially in a coordinated fashion. To investigate whether the processing reaction requires a precise spacing of the sequences directing termination and processing, the distance of both sequence elements was altered by inserting 10,22 , or $57 \mathrm{bp}$ of foreign DNA sequences, respectively, into the unique BglII site present in pMrC552-690*. Reaction conditions were chosen that allowed both termination and processing to take place. In Figure 5C, the transcripts syn- 
thesized in the cell-free system are shown. It is obvious that the size of the RNA molecules synthesized from the different templates increases by the number of nucleotides inserted between the processing and the termination site. Nuclease S1 mapping experiments (not shown) demonstrate that the $3^{\prime}$ ends of the primary transcripts derived from the push-apart mutants map 11 bp upstream of the Sal box in every case. This result demonstrates that (1) the stop of the pol I elongation reaction and, thus, the 3' end formation of the primary transcript are solely directed by the Sal box element and are independent on $5^{\prime}$-flanking sequences, and (2) that the $3^{\prime}$-terminal processing reaction, on the other hand, requires either an exact spacing of the termination and the processing signal and/or a defined RNA sequence.

\section{Discussion}

In this study we have investigated the molecular mechanisms of $3^{\prime}$-end formation of mouse pre-rRNA. Previously, we have shown that RNA pol I terminates transcription upstream of the 18-bp Sal box sequence element, which functions as a termination signal (Grummt et al. 1985, 1986; Bartsch et al. 1987; Kuhn et al. 1988). We have demonstrated that the generation of correct ends of mouse pre-rRNA is a two-step process. Both the stop of the transcription elongation reaction by RNA pol I and the release of the rDNA transcripts takes place 11 bp upstream of the Sal box termination signal, independent of the sequences surrounding this sequence element. The termination reaction is followed by a limited $3^{\prime}$-terminal trimming of the primary transcript by 10 nucleotides, yielding the $3^{\prime}$ end of the first semistable pre-rRNA, which maps 565 bp downstream of the $28 \mathrm{~S}$ RNA-coding region, i.e., 21 bp upstream of the Sal box. This processing event occurs post-transcriptionally and is dependent on the formation of correct $3^{\prime}$ ends of the primary transcripts. Nonterminated read-through transcripts generated either in the extract system or exogenous RNA substrates synthesized by SP6 RNA polymerase were not processed. This finding suggests that either the processing event is intimately coupled to the termination reaction or that the processing nuclease(s) recognize the specific $3^{\prime}$-terminal sequence or structure of pre-rRNA. The second possibility appears to be more likely. We have shown that processing can be uncoupled from the termination process provided that the RNA molecules contain the correct $3^{\prime}$ end. Terminated transcripts synthesized in vitro in a short-term reaction were specifically processed in the presence of extract protein. The participation of specific enzyme(s) in this nucleolytic cleavage reaction is demonstrated by two lines of evidence: (1) Different extracts direct different amounts of terminated and processed transcripts; (2) processing of the primary transcripts in vitro requires the presence of cellular proteins, indicating that the removal of the $3^{\prime}$ terminal nucleotides is not an autocatalytic process. The reaction does not require the supply of energy, as shown by its independence of NTPs.

At present, we have no information on the nature of the 3 '-processing factor(s) and its relation to the pol I transcription machinery. Clearly, this nucleolytic activity is not an integral part of the termination factor itself, as both activities can be separated chromatographically. The isolated termination factor TTF-I does not exhibit nucleolytic activity; however, we have preliminary experimental evidence that the processing activity may be associated with RNA pol I. Pol I that has been purified by four different columns of ion exchange chromatography (including gradient elution on two FPLC columns) still contains the processing activity (A. Kuhn and C. Schoneberg, unpubl.). Thus, the nuclease activity could reside in one of the subunits of pol I.

Both the functional significance and the molecular mechanism of this specific 3 '-terminal cleavage reaction still remains to be elucidated. In most specific cleavage reactions of pol-II-catalyzed transcripts, the involvement of small nuclear RNAs has been demonstrated (Birchmeier et al. 1984; Frendewey and Keller 1985; Hashimoto and Steitz 1986). Whether or not small nuclear ribonucleoproteins (snRNPs) also play a role in the processing of rRNA precursors is still unknown. A likely candidate for assistance in pre-rRNA processing would be the nucleolus-specific U3 RNA for which no function has yet been demonstrated. It was noted that the sequence at the extreme $5^{\prime}$ end of the internal transcribed spacer II (ITS II) was conserved among vertebrate species and was complementary to nucleotides $158-167$ of rat U3 RNA (Bachellerie et al. 1983). Therefore, it was postulated that U3 RNA may base pair to this sequence and mediate the cleavage of $5.8 \mathrm{~S}$ RNA from the ITS II. However, experimental evidence in support of this model is still lacking. In addition to this homology to the internal ribosomal gene region, a pronounced sequence complementarity exists between the 3 '-terminal region of mouse pre-rRNA (from +557 to +580 ) and nucleotides 178-199 of mouse U $3_{B}$ RNA. Therefore, it is tempting to speculate that U3 RNA participates in the 3 '-terminal processing reaction. We have performed a number of experiments to demonstrate an involvement of U3 RNP in this process. We have used U3-specific antibodies, U3 RNA in sense or anti-sense orientation, and U3-specific oligonucleotides and treated the extracts with micrococcal nuclease (data not shown). None of these treatments affected either the initiation, termination, or 3'terminal processing reaction. Thus, it is rather unlikely that an RNA component plays an essential role in any of these reactions.

The physiological relevance of this 3 '-terminal processing that specifically removes 10 bases from $>14,000$ nucleotide transcripts, as well as its relation to other nucleolytic reactions that convert the primary rDNA transcript into mature $18 \mathrm{~S}, 5.8 \mathrm{~S}$, and $28 \mathrm{~S} \mathrm{RNA}$, remains to be established. We suspect a role of this trimming reaction in pre-rRNA stability because we consistently observed both in transfection assays and in the cell-free transcription system a higher stability of specifically terminated and processed transcripts, as compared to readthrough RNA molecules. Presumably, either a protein is transferred to the $3^{\prime}$ end of the processed transcript or a 
defined structure of the RNA is created that prevents the degradation by nonspecific nucleases. The formation of either the specific $3^{\prime}$ end or the association with a putative terminal protein, in turn, could be a prerequisite for the formation of higher order structures that facilitate the interaction with other proteins involved in processing and/or ribosome assembly.

\section{Materials and methods}

\section{Plasmid constructions}

Most of the recombinant plasmids used have been described before (Kuhn et al. 1988). The artificial ribosomal minigene constructs contain sequences from -169 to +155 from the $5^{\prime}$ region of the rDNA repeat fused to $3^{\prime}$-terminal spacer fragments covering sequences from +552 to +604 (pMrC552604 ), from +552 to +650 (pMrC552-650), or from +552 to +690 (pMrC552-690), with respect to the 3 ' end of the $28 \mathrm{~S}$ RNA-coding region. To also use these constructs in transfection experiments and to facilitate nuclease S1 mapping, a 167bp reporter gene fragment (StuI-PvuII fragment from pSV2CAT) was inserted into the $A v a I$ site (at position +131 ) within the promoter fragment. The unique HindIII site present within the CAT fragment was used for S1 mapping. To construct the push-apart mutants pMrPA10, pMrPA22, and pMrPA57, the nucleotides +581 , +582 , and +585 were converted into $G, A$, and $T$ residues by oligonucleotide-directed mutagenesis, which yielded the clone pMrC552-690*. This construct is identical to pMrC552-690, except that a new BgIII site has been created at position +580 . Into this BgIII site, either a 10-bp oligonucleotide (GATCCCCGGG) or a 22-bp AluI fragment from pUC9 or a 57-bp AluI fragment from pBR322, respectively, was inserted to change the distance between the termination site and the Sal box. SP6 transcripts were generated from SP64/C552-650, a plasmid containing the 287-bp HindIII-EcoRI fragment from pMrC552-650 adjacent to the SP6 promoter.

\section{Transcription assays}

Ehrlich ascites cells were cultured in RPMI medium containing $5 \%$ newborn calf serum. S-100 extracts were prepared according to Weil et al. (1979), and nuclear extracts according to Dignam et al. (1983). Usually, cell-free transcriptions were performed in 25- $\mu$ l assays containing $15 \mathrm{ng}$ of template DNA (which was either used in the circular form or after linearization with EcoRI), $35 \mathrm{ng}$ of pUC9 carrier DNA, and $15 \mu \mathrm{l}$ of a mixture of nuclear and S-100 extract. The reaction cocktail contained $12 \mathrm{mM}$ HEPES (pH 7.9), $85 \mathrm{~mm} \mathrm{KCl,} 0.12 \mathrm{~mm}$ EDTA, $5 \mathrm{~mm} \mathrm{MgCl}_{2}, 10$ $\mathrm{mM}$ creatine phosphate, $0.6 \mathrm{mM}$ each of ATP, CTP, and UTP, $12.5 \mu \mathrm{M}$ GTP, and $1 \mu \mathrm{Ci}$ of $\left[\alpha^{-32} \mathrm{P}\right] \mathrm{GTP}$. The mixture was incubated at $30^{\circ} \mathrm{C}$ for the times indicated in the figure legends and was then processed for gel analysis (Grummt 1981). Samples to be analyzed by nuclease $S 1$ mapping were synthesized in the presence of $0.6 \mathrm{mM}$ cold GTP and treated with DNase I before phenol extraction and ethanol precipitation.

\section{Transfection of cells and preparation of cellular RNA}

Subconfluent cultures of $3 \mathrm{~T} 6$ cells were transfected with $20 \mu \mathrm{g}$ of supercoiled plasmid DNA by the calcium phosphate technique (Graham and Van der Eb 1973). The cells were harvested 44-49 hr after transfection. RNA was isolated according to Chirgwin et al. (1979), dissolved in sterile water at $2 \mathrm{mg} / \mathrm{ml}$, and used for hybridization.
Mapping of RNA 3' ends by the nuclease S1 technique

RNA from $50-\mu l$ in vitro transcription assays or $50 \mu \mathrm{g}$ of cellular RNA extracted from transfected cells was mixed with the labeled probe (HindIII-EcoRI fragment from pMrC552-650), precipitated with ethanol, and dissolved in $25 \mu$ l hybridization buffer $180 \%$ formamide, $0.4 \mathrm{M} \mathrm{NaCl}, 0.04 \mathrm{M}$ PIPES at $\mathrm{pH} 6.4$, and $1 \mathrm{mM}$ EDTA). After hybridization at $50^{\circ} \mathrm{C}$ the reactions were diluted with $250 \mu \mathrm{l}$ of nuclease S1 buffer and digested with 250 units nuclease S1 (Pharmacia) for $1 \mathrm{hr}$ at room temperature. The hybrids were purified by extraction with phenolchloroform and analyzed on $6 \%$ sequencing gels, along with size markers.

\section{Acknowledgments}

We thank Ingrid Bartsch for providing highly purified termination factor TTF-I and Wolfgang Hädelt for preparation of cell extracts. This work was supported by the Deutsche Forschungsgemeinschaft and the Fond der Chemischen Industrie.

\section{References}

Bachellerie, J.-P., B. Michot, and F. Raynal. 1983. Recognition signals for mouse pre-rRNA processing-A potential role for U3-nucleolar RNA. Mol. Biol. Rep. 9: 79-86.

Bartsch I., C. Schoneberg, and I. Grummt. 1987. Evolutionary changes of sequences and factors that direct transcription termination of human and mouse ribosomal genes. Mol. Cell. Biol. 7: 2521-252.

. 1988. Purification and characterization of TTF I, a factor that mediates termination of mouse ribosomal DNA transcription. Mol. Cell. Biol. 8: 3891-3897.

Birchmeier, C., D. Schümperli, G. Sconzo, and M.L. Birnstiel. 1984. 3' editing of mRNAs: Sequence requirements and involvement of a 60 nucleotide RNA in maturation of histone mRNA precursors. Proc. Natl. Acad. Sci. 81: 1057-1061.

Birnstiel, M.L., M. Busslinger, and K. Strub. 1985. Transcription termination and processing: The end is in site. Cell 41: 349-359.

Bogenhagen, D.F. and D.D. Brown. 1981. Nucleotide sequences in Xenopus 5S DNA required for transcription termination. Cell 24: 261-270.

Castano, J.G., J.A. Tobian, and M. Zasloff. 1985. Purification and characterization of an endonuclease from Xenopus laevis ovaries which accurately processes the $3^{\prime}$ terminus of human pre-tRNA ${ }^{\text {Met }}(\text { pre-tRNase })^{\star}$. I. Biol. Chem. 260: 9002-9008.

Chirgwin, J.M., A.E. Przybyla, R.J. MacDonald, and R.J. Rutter. 1979. Isolation of biologically active ribonucleic acid from sources enriched in ribonuclease. Biochemistry 18: 52945299.

Connelly, S. and J. Manley. 1988. A functional mRNA polyadenylation signal is required for transcription termination by RNA polymerase II. Genes Dev. 2: 440-452.

Cozzarelli, N.R., S.P. Gerrard, M. Schlissel, D.D. Brown, and D.F. Bogenhagen. 1983. Purified RNA polymerase III accurately and efficiently terminates transcription of $5 \mathrm{~S}$ RNA genes. Cell 34: 829-835.

Dignam, J.D., R.M. Lobowitz, and R.G. Roeder. 1983. Accurate transcription initiation by RNA polymerase II in a soluble extract from isolated mammalian nuclei. Nucleic Acids Res. 11: $1475-1489$.

Frendewey D. and W. Keller. 1985. Stepwise assembly of a prerRNA splicing complex requires U-snRNPs and specific intron sequences. Cell 42: 355-367. 
Frendewey, D., T. Dingermann, L. Cooley, and D. Söll. 1985. Processing of precursor tRNAs in Drosophila. J. Biol. Chem. 260: 449-454.

Graham, F.C. and A.J. Van der Eb. 1973. A new technique for the assay of infectivity of human adenovirus 5 DNA. Virology 52: 456-464.

Grummt, I. 1981. Specific transcription of mouse ribosomal DNA in a cell-free system that mimics control in vivo. Proc. Nat1. Acad. Sci. 78: 727-731.

Grummt, 1., U. Maier, A. Oehrlein, N. Hassouna, and J.-P. Bachellerie. 1985. Transcription of mouse rDNA terminates downstream of the $3^{\prime}$ end of 28 S RNA and involves the interaction of factors with repeated sequences in the $3^{\prime}$ spacer. Cell 43: 801-810.

Grummt, I., H. Rosenbauer, I. Niedermeyer, U. Maier, and A. Öhrlein. 1986. A repeated 18 bp sequence motif in the mouse rDNA spacer mediates binding of a nuclear factor and transcription termination. Cell 45: 837-846.

Hashimoto, C. and J.A. Steitz. 1986. A small ribonucleoprotein associates with the AAUAAA polyadenylation signal in vitro. Cell 45: 581-591.

Kuhn, A., A. Normann, I. Bartsch, and I. Grummt. 1988. The mouse ribosomal gene terminator consists of three functionally separable sequence elements. EMBO I. 7: 14971502.

Labhard, P. and R.H. Reeder. 1986. Characterization of three sites of RNA 3 ' end formation in the Xenopus ribosomal gene spacer. Cell 45: 431-443.

1987. A 12-base-pair sequence is an essential element of the ribosomal gene terminator in Xenopus laevis. Mol. Cell. Biol. 7: 1900-1905.

Logan, J., E. Falk-Pederson, J.E. Darnell, and T. Shenk. 1987. A poly(A) addition site and a downstream termination region are required for efficient cessation of transcription by RNA polymerase II in the mouse $\beta$-globin gene. Proc. Natl. Acad. Sci. 84: 8306-8310.

Nevins, J.R. 1983. The pathway of eukaryotic mRNA formation. Annu. Rev. Biochem. 52: 441-466.

Weil, P.A., D.S. Luse, J. Segall, and R.G. Roeder. 1979. Selective and accurate initiation of transcription at the $\mathrm{Ad} 2$ major late promoter in a soluble system dependent on purified RNA polymerase II and DNA. Cell 18: 469-484.

Whitelaw, E. and N. Proudfoot. 1986. $\alpha$-Thallassaemia caused by a poly(A) site mutation reveals that transcription termination is linked to $3^{\prime}$-end processing in the human $\alpha-2$ globin gene. EMBO J. 5: 2915-2922. 


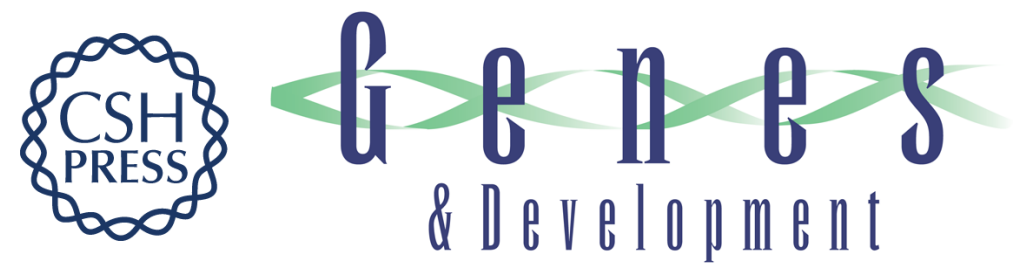

\section{3'-end formation of mouse pre-rRNA involves both transcription termination and a specific processing reaction.}

A Kuhn and I Grummt

Genes Dev. 1989, 3:

Access the most recent version at doi:10.1101/gad.3.2.224

References This article cites 25 articles, 9 of which can be accessed free at: http://genesdev.cshlp.org/content/3/2/224.full.html\#ref-list-1

License

Email Alerting

Service

Receive free email alerts when new articles cite this article - sign up in the box at the top right corner of the article or click here.

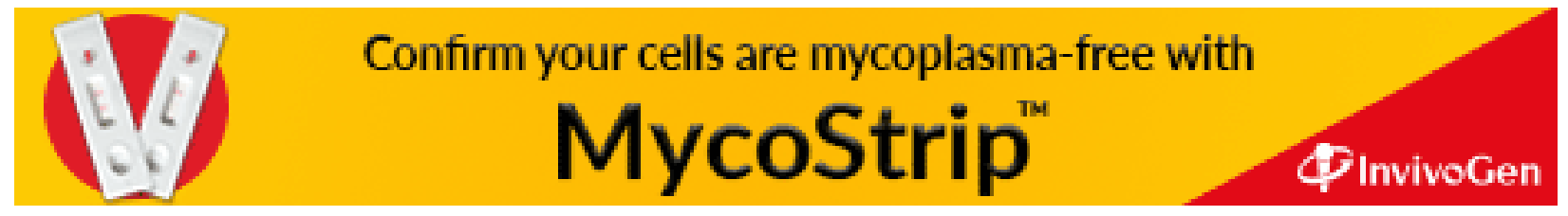

